\title{
Importance of iron deficiency in patients with chronic heart failure as a predictor of mortality and hospitalizations: insights from an observational cohort study
}

José González-Costello ${ }^{1 *} \mathbb{D}$, Josep Comín-Colet ${ }^{1}$, Josep Lupón ${ }^{3}$, Cristina Enjuanes², Marta de Antonio³, Lara Fuentes ${ }^{1}$, Pedro Moliner-Borja ${ }^{2}$, Nuria Farré ${ }^{2}$, Elisabet Zamora ${ }^{3}$, Nicolás Manito', Ramón Pujol ${ }^{4}$ and Antoni Bayés-Genis ${ }^{3}$

\begin{abstract}
Background: Iron deficiency (ID) in patients with chronic heart failure (CHF) is considered an adverse prognostic factor. We aimed to evaluate if ID in patients with CHF is associated with increased mortality and hospitalizations.

Methods: We evaluated ID in patients with CHF at 3 university hospitals. ID was defined as absolute (ferritin $<100 \mu \mathrm{g} / \mathrm{L}$ ) or functional (transferrin Saturation index $<20 \%$ and ferritin between 100 and $299 \mu \mathrm{g} / \mathrm{L}$ ). We excluded patients who received treatment with intravenous Iron or Erythropoietin during follow-up. We evaluated if ID was a predictor of death or hospitalization due to heart failure or any cause using univariate and multivariate cox regression analysis.
\end{abstract}

Results: We included 1684 patients, 65\% males, 38\% diabetics, median age of 72 years, 37\% in functional class III-IV and $30 \%$ of patients with a left ventricular ejection fraction $>45 \%$. Patients were well treated, with $87 \%$ and $88 \%$ of patients receiving renin-angiotensin inhibitors and beta-blockers, respectively. Median transferrin saturation index was 20\%, median ferritin $155 \mathrm{ng} / \mathrm{mL}$ and median haemoglobin $13 \mathrm{~g} / \mathrm{dL}$. ID was present in 53\% of patients; in 35\% it was absolute and in 18\% functional. Median follow-up was 20 months. ID was a predictor of death, hospitalization due to heart failure or to any cause in univariate analysis but not after multivariate analysis. No differences were found between absolute or functional ID regarding prognosis.

Conclusion: In a real life population of patients with CHF and a high prevalence of heart failure with preserved ejection fraction, ID did not predict mortality or hospitalizations after adjustment for comorbidities, functional class and neurohormonal treatment.

Keywords: Chronic heart failure, Iron deficiency, Mortality, Hospitalization

\section{Background}

Iron deficiency (ID) affects up to $50 \%$ of patients with chronic heart failure (HF) and has been identified as an adverse prognostic factor independently of the presence of anaemia and chronic kidney disease (CKD) [1, 2]. However, in another study of community-dwelling adults with self-reported heart failure there was no association

\footnotetext{
* Correspondence: jgcostello@hotmail.com

${ }^{1}$ Area de Enfermedades del Corazón, Hospital Universitari de Bellvitge,

IDIBELL, Universitat de Barcelona, L'Hospitalet de Llobregat, Feixa Llarga SN, 08907 Barcelona, Spain

Full list of author information is available at the end of the article
}

between ID and all cause or cardiovascular mortality, but haemoglobin and C-reactive protein did predict worse survival [3]. ID frequently overlaps with anaemia and/or CKD in chronic HF and the presence of ID amplifies mortality risk, either alone or in combination with anaemia, CKD, or both [2].

Although the pathophysiology behind ID in patients with chronic HF is not fully understood and is considered multifactorial [4-6], treatment of ID in these patients has become a therapeutic goal. Oral iron is not associated with an increase in exercise capacity in patients with HF and systolic dysfunction [7]. Recent randomized trials have 
demonstrated how intravenous iron can improve exercise capacity, cardiac function, symptom severity and quality of life in patients with chronic HF and left ventricular systolic dysfunction [8-11]. In the most recent study, the CONFIRM-HF, the risk of hospitalization due to worsening heart failure was reduced in the ferric carboxymaltose arm vs. the placebo-treated arm. However, no study has evaluated the incidence of hospitalizations due to heart failure or to any cause in patients with chronic HF and ID.

Given the importance of reducing mortality and hospitalizations in patients with chronic HF and that treatment with intravenous iron may reduce hospitalizations, we aimed to evaluate the prognostic importance of ID in a large multicenter cohort of ambulatory patients with chronic HF, focusing on mortality and hospitalizations due to worsening heart failure or to any cause.

\section{Methods}

\section{Study design}

This is a retrospective observational cohort study of patients with heart failure in whom we evaluated the presence of ID and whether ID was associated with re-hospitalizations or mortality during follow-up.

\section{Component population}

The study population consisted of 2495 consecutive patients with stable chronic HF prospectively enrolled at 3 multidisciplinary heart failure (HF) units from 3 tertiary hospitals in Spain from 2005 to 2012. Patients had to be diagnosed of HF according to the European Society of Cardiology diagnostic criteria [12]. We defined HF with preserved LVEF (HFPEF) as the presence of symptoms of HF with a left ventricular ejection fraction $\geq 50 \%$ and an objective evidence of cardiac dysfunction (enlarged left atrium, diastolic dysfunction or elevated natriuretic peptides). We excluded patients without iron status assessed at entry and those with significant primary valvular or pericardial disease. Of the remaining 2172 patients, we excluded those who received treatment with intravenous iron or subcutaneous erythropoetin at any time point during follow-up, after review of the pharmacy records, as this treatment could influence outcomes. This left 1684 patients for analysis. Patients were treated according to the European Society of Cardiology guidelines [12].

Written informed consent was obtained from each patient. The investigation conforms with the principles outlined in the Declaration of Helsinki. The local ethic committees approved the study protocol.

\section{Pooled methodology}

The pooled data for the present study were assessed at a patient level and collected prospectively. The end points for the present study were all cause mortality, first hospitalization due to HF and first hospitalization due to any cause after inclusion in the HF unit. Vital status and hospitalizations were assessed by review of the clinical databases, hospital records or direct contact with patients or relatives. Follow-up duration was until death or up to the last visit in the out patient clinic before October 2015. For patients lost to follow-up, we contacted the patient or the family by telephone and determined vital status and hospitalizations. For patients that could not be contacted by telephone we accessed the electronic clinical history of the hospital and of the primary care health system and determined vital status and hospitalizations. If despite all these efforts, no information regarding hospitalizations and death could be obtained, end of follow-up was determined to be the last time the patient had been to the out patient clinic. Five patients received a heart transplant during follow-up and this was recorded as death and end of follow-up. No patient received a ventricular assist device.

\section{Iron status and other laboratory measurements}

The following blood biomarkers reflecting iron status were measured at study entry: Serum iron (ug/dL) was measured using spectrophotometry, serum ferritin $(\mathrm{ng} / \mathrm{mL})$ and transferrin $(\mathrm{mg} / \mathrm{dL})$ were measured using immunoturbidimetry. Transferrin saturation index (TSAT) was estimated using the formula: $\mathrm{TSAT}=$ serum iron $(\mathrm{ug} / \mathrm{dL}) /[$ serum transferrin $(\mathrm{mg} / \mathrm{dL}) \times 1.25$ ] [13]. ID was defined as absolute when ferritin $<100 \mathrm{ng} / \mathrm{ml}$ and functional when TSAT $<20 \%$ with ferritin 100-299 $\mathrm{ng} / \mathrm{ml}[8]$.

Haemoglobin was measured using impedance laser colorimetry and anaemia was defined as Haemoglobin < $12 \mathrm{~g} / \mathrm{dL}$ in women and Haemoglobin $<13 \mathrm{~g} / \mathrm{dL}$ in men. Concentrations of $\mathrm{N}$-terminal pro-brain-type natriuretic peptide (NT-proBNP) were measured using an immunoassay based on electrochemiluminescence on the Elecsys



Fig. 1 Flowchart of the study population. 105 of patients treated with erythropoietin were also treated with intravenous iron 
System (Roche Diagnostics, Basel, Switzerland). Renal function was assessed with the estimated glomerular filtration rate using the abbreviated Modification of Diet in Renal Disease equation.

\section{Statistical analysis}

Continuous variables were explored for normal distribution according to histograms and the Shapiro-Wilk test. The comparison of quantitative variables between the 2 groups was done using the Student-t test for normally distributed variables and the Mann-Whitney $U$ test for non-normally distributed variables. The chi-square test was used for categorical variables and Fisher's exact test when cells had an expected count of less than 5 .

The continuous variables that had a very skewed distribution, such as the TSAT, Ferritin and NT-proBNP underwent logarithmic transformation. The resulting variables are referred to as: $\log$ TSAT, $\log$ Ferritin and $\operatorname{logNT}$-proBNP. In order to assess if variables introduced in the multivariate regression analysis were highly correlated we evaluated collinearity using the variance inflation factor. The maximal variance inflation factor found

Table 1 Baseline variables according to iron deficiency

\begin{tabular}{|c|c|c|c|c|}
\hline Variables & Overall $N=1684$ & No ID $N=786$ & ID N $=898$ & $P$ value \\
\hline Age (years) & $72(61-79)$ & 70 (58-78) & 73 (63-79) & $<0.0001$ \\
\hline Female gender & $588(35)$ & $207(26)$ & $381(42)$ & $<0.0001$ \\
\hline $\mathrm{BMI}\left(\mathrm{kg} / \mathrm{m}^{2}\right)$ & $27(24-30)$ & $30(27-34)$ & $31(27-36)$ & 0.011 \\
\hline Systolic BP (mm Hg) & $120(108-139)$ & $118(105-133)$ & $125(110-140)$ & $<0.0001$ \\
\hline Heart rate (bpm) & $70(62-80)$ & $70(60-80)$ & $70(62-80)$ & 0.398 \\
\hline NYHA Class III-IV & $592(35)$ & $218(28)$ & $374(42)$ & $<0.0001$ \\
\hline LVEF (\%) & $35(27-50)$ & $35(27-48)$ & $36(28-53)$ & 0.032 \\
\hline HFPEF & $428(25)$ & $182(23)$ & $246(27)$ & 0.046 \\
\hline Ischaemic aetiology & $738(44)$ & $331(42)$ & $407(45)$ & 0.185 \\
\hline Hypertension & $1136(68)$ & $492(63)$ & $644(72)$ & $<0.0001$ \\
\hline Diabetes Mellitus & $639(38)$ & $262(33)$ & $377(42)$ & $<0.0001$ \\
\hline COPD & $318(19)$ & $158(20)$ & $160(18)$ & 0.232 \\
\hline \multicolumn{5}{|l|}{ Treatment } \\
\hline ACE-I or ARB & $1457(87)$ & $695(89)$ & $762(85)$ & 0.019 \\
\hline Beta blockers & $1474(88)$ & $690(88)$ & $784(87)$ & 0.766 \\
\hline MRAs & $716(43)$ & $357(45)$ & $359(40)$ & 0.024 \\
\hline Digoxin & $395(23)$ & $184(23)$ & $211(24)$ & 0.966 \\
\hline Oral anticoagulation & $755(45)$ & $356(45)$ & $399(44)$ & 0.723 \\
\hline Antiplatelet & $879(52)$ & $412(52)$ & $467(52)$ & 0.884 \\
\hline Diuretics & $1458(87)$ & $661(84)$ & 797 (89) & 0.001 \\
\hline ICD & $136(8)$ & $64(8)$ & $72(8)$ & 0.933 \\
\hline Resynchronization & $77(5)$ & $35(5)$ & $42(5)$ & 0.826 \\
\hline \multicolumn{5}{|l|}{ Laboratory values } \\
\hline Haemoglobin (g/dL) & $13.0(11.7-14.3)$ & $13.4(12.0-14.7)$ & $12.7(11.5-13.9)$ & $<0.0001$ \\
\hline eGFR (ml/min/1.73 m²) & $53(37-71)$ & $56(38-75)$ & $52(36-68)$ & 0.032 \\
\hline Ferritin (ng/mL) & $155(72-276)$ & $276(172-435)$ & $76(43-141)$ & $<0.0001$ \\
\hline Transferrin (mg/dL) & $261(224-313)$ & $245(213-288)$ & $276(239-330)$ & $<0.0001$ \\
\hline Serum iron (ug/dL) & $73(51-99)$ & $90(71-116)$ & $59(44-79)$ & $<0.0001$ \\
\hline TSAT (\%) & $20(15-28)$ & $26(22-34)$ & $16(12-19)$ & $<0.0001$ \\
\hline NTproBNP (ng/L) & $1355(536-3171)$ & $1111(488-2747)$ & $1584(609-3597)$ & $<0.0001$ \\
\hline Sodium (mmol/L) & $140(138-142)$ & $140(138-142)$ & $140(138-142)$ & 0.011 \\
\hline
\end{tabular}

Continuous variables are expressed as median and interquartile range (IQR). Categorical variables are expressed as number and percentage ID Iron deficiency, BMI Body mass index, NYHA New York Heart Association, LVEF Left ventricular ejection fraction, HFPEF Heart failure with preserved ejection fraction, COPD Chronic obstructive pulmonary disease, ACE-I Angiotensin converting enzyme inhibitor, ARB Angiotensin II receptor blocker, MRA Mineralocorticoid receptor antagonist, ICD Implantable cardioverter defibrillator, eGFR estimated glomerular filtration rate, TSAT Transferrin saturation, NTproBNP N-terminal pro-brain natriuretic peptide 
among baseline variables was 1.5 , indicating a low degree of collinearity.

To evaluate predictors of ID we used multivariate logistic regression analysis. All baseline variables with a significant univariate association $(p<0.10)$ or deemed to be clinically relevant were entered in a step-wise backward multivariable model with an exclusion criteria of $p>0.10$. Additional bootstrap analysis (1000 cycles) of the multivariate model was performed to measure accuracy of the estimated model. The R2 value for the multivariate model was also calculated.

Incidence ratio of death and hospitalizations due to $\mathrm{HF}$ or any cause were determined dividing the incidence rate of events in patients with ID vs. without ID. Significance was assessed using the Mantel-Haenszel Chi square test.

Survival and time to first hospitalization due to heart failure or any cause were evaluated using Kaplan Meier survival curves. We evaluated if ID was a predictor of survival or hospitalization using univariate Cox proportional hazard regression analysis. With the variables that were significant in the univariate analysis $(p<0.10)$ and those that were deemed to be clinically relevant, we then performed backward step multivariate Cox proportional hazard analysis with an exclusion criteria of $p>0.10$. ID is forced in to the final multivariate model given that it is the purpose of our study. The proportionality assumption for the Cox regression analysis was evaluated using residual analysis. Significance was set at $p<0.05$ (2 tailed) and SPSS version 18 and Stata 14 were used to perform all statistical evaluations.

\section{Results}

We included 1684 patients in the analysis (see flowchart in Fig. 1), whose baseline characteristics are shown in Table 1. ID was present at inclusion in 898 (53\%) patients. In $35 \%$ it was absolute ID and in $18 \%$ functional ID. There were 953 (57\%) patients who were not

Table 2 Predictors of iron deficiency using logistic regression analysis

\begin{tabular}{|c|c|c|c|c|}
\hline Variables & Univariate OR & $P$ value & Multivariate OR & $P$ value \\
\hline Age (per 5 years) & $1.09(1.05-1.13)$ & $<0.001$ & & \\
\hline Female sex & $2.05(1.67-2.53)$ & 0.396 & $1.62(1.29-2.03)$ & $<0.001$ \\
\hline Hypertension & $1.50(1.23-1.85)$ & $<0.001$ & & \\
\hline Diabetes Mellitus & $1.44(1.18-1.76)$ & $<0.001$ & & \\
\hline COPD & $0.86(0.67-1.10)$ & 0.223 & & \\
\hline HFPEF & $1.25(1.00-1.56)$ & 0.047 & & \\
\hline Ischaemic aetiology & $1.15(0.94-1.39)$ & 0.175 & & \\
\hline NYHA class III-IV & $1.84(1.50-2.26)$ & $<0.001$ & $1.50(1.20-1.88)$ & $<0.001$ \\
\hline Systolic BP (per 10 mmHg) & $1.11(1.06-1.16)$ & $<0.001$ & $1.09(1.04-1.14)$ & $<0.001$ \\
\hline Heart rate (per 10 bpm) & $1.03(0.97-1.10)$ & 0.379 & & \\
\hline BMI $\left(1 \mathrm{~kg} / \mathrm{m}^{2}\right)$ & $1.03(1.01-1.04)$ & 0.008 & $1.03(1.00-1.05)$ & 0.018 \\
\hline ACE-I or ARB & $0.71(0.53-0.95)$ & 0.021 & & \\
\hline Beta blockers & $0.96(0.72-1.28)$ & 0.781 & & \\
\hline Diuretics & $1.50(1.13-1.98)$ & 0.005 & & \\
\hline MRAs & $0.81(0.66-0.98)$ & 0.028 & & \\
\hline Digoxin & $1.00(0.80-1.26)$ & 0.988 & & \\
\hline Oral anticoagulants & $0.96(0.79-1.17)$ & 0.690 & & \\
\hline Antiplatelet & $0.99(0.82-1.20)$ & 0.921 & & \\
\hline ICD & $1.02(0.71-1.44)$ & 0.94 & & \\
\hline Haemoglobin (per 1 g/dL) & $0.82(0.78-0.87)$ & $<0.001$ & $0.87(0.82-0.92)$ & $<0.001$ \\
\hline Log NT-proBNP (per 1 SD) & $1.20(1.09-1.33)$ & $<0.001$ & $1.12(0.99-1.25)$ & 0.051 \\
\hline Sodium (per 5 mmol/L) & $1.17(1.02-1.34)$ & 0.030 & & \\
\hline eGFR (per $5 \mathrm{ml} / \mathrm{min} / 1.73 \mathrm{~m}^{2}$ ) & $0.98(0.96-0.99)$ & 0.016 & & \\
\hline
\end{tabular}

OR Odds Ratio, COPD Chronic Obstructive Pulmonary Disease, HFPEF Heart failure with preserved ejection fraction, NYHA New York Heart Association, BP Blood pressure, $B M I$ Body mass index, $A C E-I$ Angiotensin converting enzyme inhibitor, ARB Angiotensin II receptor blocker, MRAs Mineralocorticoid receptor antagonists, ICD Implantable cardioverter defibrillator, Log Logarithmic transformation, NTproBNP N-terminal pro-brain natriuretic peptide, SD Standard deviation, eGFR Estimated glomerular filtration rate

Variables introduced in the multivariate model were: Age, gender, hypertension, diabetes mellitus, COPD, HFPEF, NYHA functional class III-IV, systolic BP, heart rate, $\mathrm{BMI}$, haemoglobin, logNT-proBNP, eGFR, sodium, treatment with beta blockers, ACE-i/ARBs, diuretics and MRAs 
anaemic and ID was present in $460(48 \%)$ of these. Of the 731 patients with anaemia, ID was present in 438 (60\%).

Predictors of ID after multivariate analysis are shown in Table 2. The R2 for the multivariate model was 0.05 (5\%). Median follow-up was 20 (interquartile range: 12 47) months.

\section{Iron deficiency and mortality}

Patients with ID had 307 (34\%) deaths with an incidence rate of 0.134 per year compared with those without ID, who had 212 (27\%) deaths, with an Incidence Rate of 0.101 per year. The incidence ratio was 1.32 (95\% confidence interval: $1.11-1.57 ; p=0.0017)$. Both absolute and functional ID had similar mortality rates of 0.145 and 0.128 per year, respectively, $p=0.29$. When we analysed both components of the definition of ID, lower logTSAT predicted mortality but not logFerritin. ID was not a predictor of mortality in multivariate analysis. Table 3 shows the hazard ratios for mortality in our cohort of patients using univariate and multivariate cox regression analysis. When we introduced logTSAT in the multivariate model, instead of the usual definition of ID, it also was not a significant predictor of mortality. Figure 2 shows no differences in the survival curves stratified for ID after adjustment for the covariates that were significant in the multivariate model.

\section{Iron deficiency and hospitalizations due to heart failure}

Patients with ID had 246 (27\%) hospitalizations due to HF with an incidence rate of 0.121 per year compared with those without ID, who had 182 (23\%) hospitalizations due HF, with an incidence rate of 0.097 per year. The incidence ratio was 1.24 (95\% confidence interval: $1.03-1.51 ; p=0.025)$. There was no significant difference in the incidence rate of hospitalizations due to heart failure between patients with functional or absolute ID: 0.110 and 0.125 per year respectively, $p=0.37$. ID was not a predictor of hospitalization due to heart failure after multivariate analysis. Table 4 shows predictors of hospitalization due to HF after univariate and multivariate adjustment. Figure 3 shows the survival curves for

Table 3 Predictors of mortality using cox regression analysis

\begin{tabular}{|c|c|c|c|c|}
\hline Variables & Univariate HR & $P$ value & Multivariate HR & $P$ value \\
\hline Age (per 5 years) & $1.36(1.30-1.42)$ & $<0.001$ & $1.21(1.16-1.27)$ & $<0.001$ \\
\hline Female sex & $1.08(0.90-1.30)$ & 0.39 & $0.65(0.54-0.79)$ & $<0.001$ \\
\hline Hypertension & $1.35(1.12-1.63)$ & 0.002 & & \\
\hline Diabetes Mellitus & $1.51(1.27-1.80)$ & $<0.001$ & $1.30(1.08-1.55)$ & 0.005 \\
\hline COPD & $1.63(1.33-1.99)$ & $<0.001$ & & \\
\hline HFPEF & $1.11(0.90-1.36)$ & 0.34 & & \\
\hline Ischaemic aetiology & $1.09(0.92-1.30)$ & 0.33 & & \\
\hline NYHA class III-IV & $3.14(2.63-3.74)$ & $<0.001$ & $1.85(1.53-2.24)$ & $<0.001$ \\
\hline Systolic BP (per 10 mmHg) & $0.97(0.93-1.01)$ & 0.112 & & \\
\hline Heart rate (per 10 bpm) & $1.09(1.03-1.16)$ & 0.004 & & \\
\hline BMI $\left(1 \mathrm{~kg} / \mathrm{m}^{2}\right)$ & $0.96(0.95-0.98)$ & $<0.001$ & & \\
\hline ACE-I or ARB & $0.37(0.29-0.46)$ & $<0.001$ & $0.70(0.55-0.89)$ & 0.004 \\
\hline Beta blockers & $0.41(0.33-0.51)$ & $<0.001$ & $0.59(0.47-0.74)$ & $<0.001$ \\
\hline Diuretics & $2.46(1.77-3.42)$ & $<0.001$ & $1.66(1.19-2.31)$ & 0.005 \\
\hline MRAs & $0.97(0.82-1.16)$ & 0.77 & & \\
\hline Digoxin & $1.26(1.05-1.52)$ & 0.015 & & \\
\hline ICD & $0.72(0.52-0.98)$ & 0.039 & & \\
\hline Iron deficiency & $1.32(1.11-1.57)$ & 0.002 & $1.09(0.91-1.31)$ & 0.337 \\
\hline Haemoglobin (per $1 \mathrm{~g} / \mathrm{dL}$ ) & $0.78(0.75-0.82)$ & $<0.001$ & $0.90(0.85-0.95)$ & $<0.001$ \\
\hline Log NT-proBNP (per 1 SD) & $1.93(1.76-2.11)$ & $<0.001$ & $1.49(1.34-1.66)$ & $<0.001$ \\
\hline Sodium (per 5 mmol/L) & $0.78(0.69-0.88)$ & $<0.001$ & $0.96(0.94-0.99)$ & 0.003 \\
\hline eGFR (per $5 \mathrm{ml} / \mathrm{min} / 1.73 \mathrm{~m}^{2}$ ) & $0.89(0.87-0.91)$ & $<0.001$ & & \\
\hline
\end{tabular}

HR Hazard Ratio, COPD Chronic Obstructive Pulmonary Disease, HFPEF Heart failure with preserved ejection fraction, NYHA New York Heart Association, BP Blood pressure, $B M I$ Body mass index, $A C E-I$ Angiotensin converting enzyme inhibitor, ARB Angiotensin II receptor blocker, MRAs Mineralocorticoid receptor antagonists, ICD Implantable cardioverter defibrillator, Log Logarithmic transformation, NTproBNP N-terminal pro-brain natriuretic peptide, SD Standard deviation, eGFR Estimated glomerular filtration rate

Variables introduced in the multivariate model were: Age, gender, ischaemic aetiology, systolic BP, HFPEF, diabetes mellitus, hypertension, COPD, BMI, NYHA functional class III-IV, haemoglobin, logNT-proBNP, eGFR, sodium, heart rate, treatment with beta blockers, ACE-i/ARBs, diuretics, digoxin and an ICD 


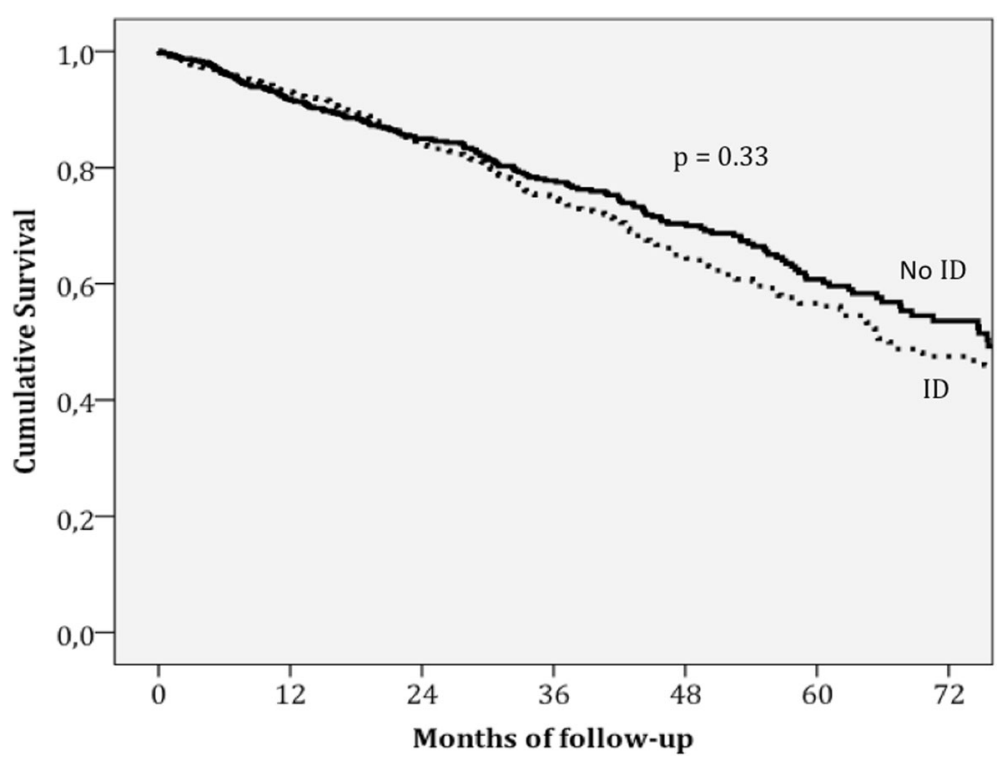

Fig. 2 Survival curves stratified for ID after adjustment for the covariates that are significant in the multivariate model: Age, sex, diabetes mellitus, New York Heart Association class III-IV, haemoglobin, logarithmic transformation of N-terminal pro-brain natriuretic peptide, serum sodium, and treatment with an angiotensin converting enzyme inhibitor or angiotensin II receptor blocker, beta blockers and diuretics

Table 4 Predictors of heart failure hospitalization using Cox regression analysis

\begin{tabular}{|c|c|c|c|c|}
\hline Variables & Univariate HR & $P$ value & Multivariate HR & $P$ value \\
\hline Age (per 5 years) & $1.23(1.18-1.29)$ & $<0.001$ & $1.11(1.05-1.17)$ & $<0.001$ \\
\hline Female sex & $1.50(1.25-1.83)$ & $<0.001$ & & \\
\hline Hypertension & $1.47(1.19-1.81)$ & $<0.001$ & & \\
\hline Diabetes Mellitus & $1.46(1.20-1.77)$ & $<0.001$ & $1.26(1.03-1.54)$ & 0.026 \\
\hline COPD & $1.76(1.41-2.19)$ & $<0.001$ & $1.63(1.28-2.07)$ & $<0.001$ \\
\hline HFPEF & $1.60(1.30-1.97)$ & $<0.001$ & $1.67(1.31-2.18)$ & $<0.001$ \\
\hline Ischaemic aetiology & $1.14(0.95-1.38)$ & 0.166 & $1.40(1.12-1.73)$ & 0.003 \\
\hline NYHA class III-IV & $2.55(2.10-3.09)$ & $<0.001$ & $1.64(1.33-2.03)$ & $<0.001$ \\
\hline Systolic BP (per 10 mmHg) & $0.97(0.93-1.01)$ & 0.123 & & \\
\hline Heart rate (per 10 bpm) & $1.09(1.03-1.16)$ & 0.005 & & \\
\hline BMI (per $\left.1 \mathrm{~kg} / \mathrm{m}^{2}\right)$ & $0.99(0.97-1.01)$ & 0.21 & & \\
\hline$A C E-I$ or $A R B$ & $0.47(0.37-0.61)$ & $<0.001$ & & \\
\hline Beta blockers & $0.46(0.36-0.58)$ & $<0.001$ & $0.71(0.54-0.93)$ & 0.013 \\
\hline Diuretics & $3.47(2.28-5.28)$ & $<0.001$ & $2.53(1.64-3.91)$ & $<0.001$ \\
\hline MRAs & $1.10(0.91-1.33)$ & 0.327 & & \\
\hline Digoxin & $1.13(0.91-1.40)$ & 0.263 & & \\
\hline$I C D$ & $0.91(0.66-1.27)$ & 0.581 & & \\
\hline Iron deficiency & $1.21(1.00-1.47)$ & 0.047 & $0.96(0.78-1.17)$ & 0.677 \\
\hline Haemoglobin (per $1 \mathrm{~g} / \mathrm{dL}$ ) & $0.84(0.80-0.89)$ & $<0.001$ & & \\
\hline Log NT-proBNP (per 1 SD) & $2.00(1.87-2.15)$ & $<0.001$ & $1.49(1.32-1.68)$ & $<0.001$ \\
\hline Sodium (per $5 \mathrm{mmol} / \mathrm{L}$ ) & $0.93(0.81-1.07)$ & 0.328 & & \\
\hline eGFR (per $5 \mathrm{ml} / \mathrm{min} / 1.73 \mathrm{~m}^{2}$ ) & $0.95(0.93-0.97)$ & $<0.001$ & & \\
\hline
\end{tabular}

HR Hazard Ratio, COPD Chronic Obstructive Pulmonary Disease, HFPEF Heart failure with preserved ejection fraction, NYHA New York Heart Association, BP Blood pressure, $B M I$ Body mass index, ACE-I Angiotensin converting enzyme inhibitor, ARB Angiotensin II receptor blocker, MRAs Mineralocorticoid receptor antagonists, ICD Implantable cardioverter defibrillator, Log Logarithmic transformation, NTproBNP N-terminal pro-brain natriuretic peptide, SD Standard deviation, eGFR Estimated glomerular filtration rate

Variables introduced in the multivariate model were gender, age, diabetes mellitus, hypertension, BMI, ischaemic aeetiology, systolic blood pressure, COPD, HFPEF, heart rate, NYHA functional class III-IV, haemoglobin, logNT-proBNP, eGFR and treatment with ACE-i/ARBs, beta blockers, diuretics and MRAs 


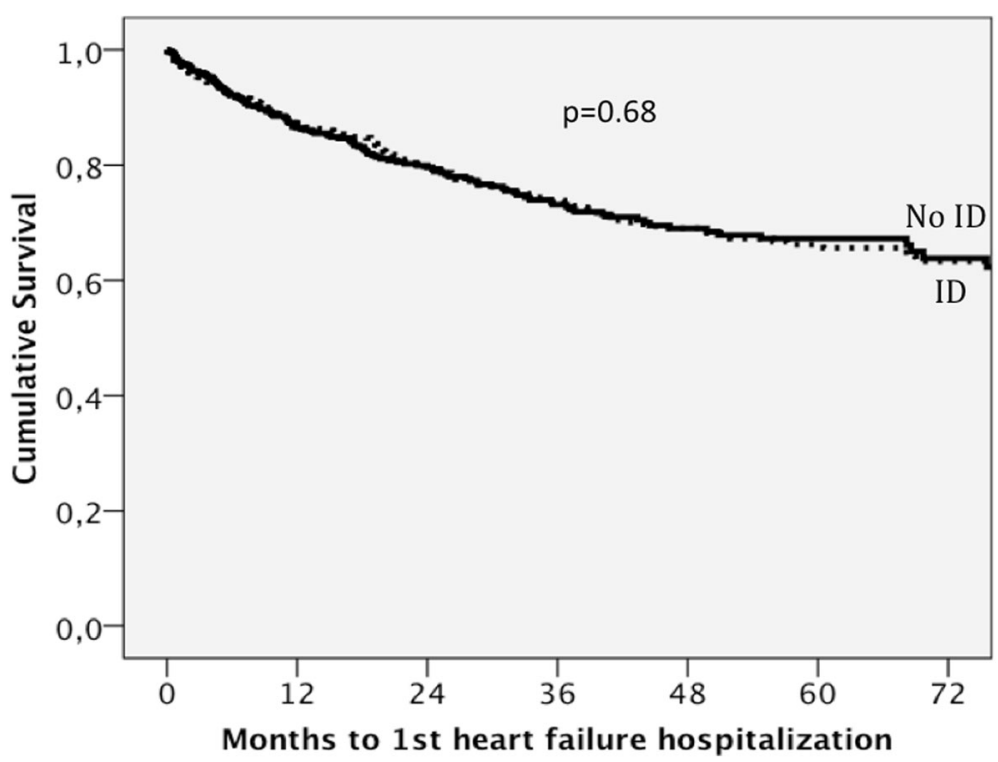

Fig. 3 Survival curves for hospitalization due to HF stratified for ID after adjustment for the covariates that are significant in the multivariate model: Age, diabetes mellitus, chronic pulmonary obstructive disease, heart failure with preserved ejection fraction, ischaemic aetiology, New York Heart Association class III-IV, logarithmic transformation of N-terminal pro-brain natriuretic peptide, and treatment with an angiotensin converting enzyme inhibitor or angiotensin II receptor blocker, beta blockers and diuretics

hospitalization due to HF stratified for ID and adjusted for the covariates that were significant in the multivariate model.

\section{Iron deficiency and hospitalizations due to any cause} Patients with ID had 465 (52\%) hospitalizations due to any cause with an Incidence Rate of 0.284 per year compared with those without ID, who had 363 (46\%) hospitalizations due to any cause, with an incidence rate of 0.226 per year. The incidence ratio was 1.26 (95\% confidence interval: $1.09-1.44 ; p=0.001$ ).

Patients with functional ID had a rate of hospitalizations due to any cause of 0.266 per year compared with 0.290 per year in those with absolute ID, $p=0.36$. ID was not a predictor of hospitalization due to any cause after multivariate analysis. Table 5 shows the predictors of hospitalization due to any cause after univariate and multivariate adjustment. Figure 4 shows the survival curves for hospitalization due to any cause stratified for ID and adjusted for the covariates that were significant in the multivariate model.

\section{Discussion}

The main findings of our study are:

1. ID was present in more than half of a large cohort of ambulatory patients with chronic HF.

2. ID did not predict mortality after multivariate adjustment.
3. ID did not predict increased hospitalizations due to heart failure or due to any cause after multivariate adjustment.

ID was very prevalent in our cohort of patients with chronic HF and most of it was due to absolute ID. Predictors of ID after multivariate analysis were female gender, a higher body mass index, more advanced functional class, increased systolic blood pressure and lower haemoglobin values. These findings are comparable to previous findings from other groups $[1-3,14]$.

\section{Iron deficiency and mortality}

ID has been shown to be a marker of increased mortality independently of anaemia and CKD [1, 2, 14], but in our study we were not able to demonstrate it after multivariate analysis. However haemoglobin remained a significant predictor of mortality, as in the study by Parikh et al. [3]. We can only hypothesize why this is, but one major difference is that in our study the number of patients with HFPEF was significant compared with none in the study by Jankowska et al. [1] and 13\% in the study by Klip et al. [14]. In the study by Parikh et al. [3] this data is unknown but given the relatively high mean systolic blood pressure, large proportion of women and age of the population it is likely that many of the participants had HFPEF. When we analyzed if ID was a predictor of mortality only in patients with systolic dysfunction, in our cohort it was not significant in 
Table 5 Predictors of hospitalization due to any cause using Cox regression analysis

\begin{tabular}{|c|c|c|c|c|}
\hline Variables & Univariate HR & $P$ value & Multivariate HR & $P$ value \\
\hline Age (per 5 years) & $1.17(1.13-1.20)$ & $<0.001$ & $1.06(1.02-1.09)$ & 0.001 \\
\hline Female sex & $1.29(1.12-1.45)$ & $<0.001$ & & \\
\hline Hypertension & $1.42(1.22-1.65)$ & $<0.001$ & & \\
\hline Diabetes Mellitus & $1.46(1.27-1.68)$ & $<0.001$ & $1.23(1.06-1.42)$ & 0.006 \\
\hline COPD & $1.56(1.32-1.84)$ & $<0.001$ & $1.44(1.21-1.71)$ & $<0.001$ \\
\hline HFPEF & $1.40(1.20-1.63)$ & $<0.001$ & $1.44(1.21-1.73)$ & $<0.001$ \\
\hline Ischaemic aetiology & $1.16(1.01-1.33)$ & 0.032 & $1.32(1.14-1.54)$ & $<0.001$ \\
\hline NYHA class III-IV & $2.03(1.77-2.34)$ & $<0.001$ & $1.40(1.20-1.64)$ & $<0.001$ \\
\hline Systolic BP (per 10 mmHg) & $1.01(0.98-1.04)$ & 0.522 & & \\
\hline Heart rate (per 10 bpm) & $1.13(1.08-1.19)$ & $<0.001$ & $1.07(1.02-1.12)$ & 0.006 \\
\hline BMI (per $\left.1 \mathrm{~kg} / \mathrm{m}^{2}\right)$ & $0.99(0.97-0.99)$ & 0.033 & & \\
\hline ACE-I or ARB & $0.50(0.41-0.61)$ & $<0.001$ & $0.80(0.65-0.98)$ & 0.030 \\
\hline Beta blockers & $0.51(0.43-0.62)$ & $<0.001$ & $0.69(0.57-0.86)$ & 0.001 \\
\hline Diuretics & $1.70(1.36-2.12)$ & $<0.001$ & $1.31(1.04-1.65)$ & 0.022 \\
\hline MRAs & $0.95(0.83-1.09)$ & 0.488 & & \\
\hline Digoxin & $1.01(0.86-1.18)$ & 0.940 & & \\
\hline ICD & $0.88(0.69-1.12)$ & 0.284 & & \\
\hline Iron deficiency & $1.21(1.05-1.39)$ & 0.007 & $0.99(0.86-1.14)$ & 0.857 \\
\hline Haemoglobin (per 1 g/dL) & $0.85(0.81-0.88)$ & $<0.001$ & $0.94(0.90-0.98)$ & 0.002 \\
\hline LogNT-proBNP (per 1 SD) & $1.53(1.42-1.64)$ & $<0.001$ & $1.31(1.20-1.42)$ & $<0.001$ \\
\hline Sodium (per 5 mmol/L) & $0.89(0.80-0.98)$ & 0.023 & & \\
\hline eGFR (per $5 \mathrm{ml} / \mathrm{min} / 1.73 \mathrm{~m}^{2}$ ) & $0.95(0.94-0.96)$ & $<0.001$ & & \\
\hline
\end{tabular}

HR Hazard Ratio, COPD Chronic Obstructive Pulmonary Disease, HFPEF Heart failure with preserved ejection fraction, NYHA New York Heart Association, BP Blood pressure, bpm beats per minute, $B M I$ Body mass index, $A C E-I$ Angiotensin converting enzyme inhibitor, ARB Angiotensin II receptor blocker, MRAs Mineralocorticoid receptor antagonists, ICD Implantable cardioverter defibrillator, Log Logarithmic transformation, NTproBNP N-terminal pro-brain natriuretic peptide, SD Standard deviation, eGFR Estimated glomerular filtration rate

We included the following variables in the multivariate analysis: Haemoglobin, sex, age, HFPEF, BMI, Diabetes Mellitus, Hypertension, COPD, ischaemic aetiology, logNTproBNP, eGFR, serum sodium, systolic BP, heart rate, NYHA functional class III-IV and treatment with ACE-I/ARB, Beta-blockers and diuretics

multivariate analysis. Our patients were more likely to be older and had more CKD and anaemia compared to those studied in the 3 previously mentioned cohorts and this may imply that other factors besides ID may be more important in predicting mortality in our patients. The variables that predicted mortality in our patients are otherwise not different from those previously described in the literature $[9,15]$.

We used the definition of ID according to the FAIR-HF trial [8]. However, diagnosis based on TSAT and ferritin may be inadequate in advanced disease or acute HF, where ferritin becomes an unreliable marker [16]. In our cohort of ambulatory patients with chronic HF, ferritin did not predict mortality in univariate analysis and the driver of adverse prognosis was the TSAT.

\section{Iron deficiency and hospitalizations}

Hospitalizations due to HF are frequent in patients with chronic HF and linked with increased mortality [17], impairment of patient's quality of life and an economic burden for the health system [18]. Although ID has been shown to be a key determinant of health-related quality of life in patients with chronic HF [19], we are not aware that the association between ID and increased risk of hospitalizations due to HF or due to any cause has been studied in patients with chronic HF. Our study is the first to do so, and we have shown that ID was associated with hospitalizations due to HF in univariate analysis but not after multivariate analysis. When we looked at predictors of hospitalization due to HF we found that they were very similar to those that predicted mortality. The only difference being that lower serum sodium and lower haemoglobin predicted mortality but not hospitalizations due to HF. On the other hand, chronic obstructive pulmonary disease, HFPEF and ischaemic etiology were associated with increased risk of hospitalization due to HF but not with mortality. Patients with chronic HF such as those seen in our study and in recent registries have many comorbidities [20], and hospitalizations may be related to these comorbidities. Therefore, we thought it would be interesting to look at hospitalizations due to any cause. ID was a predictor of hospitalizations due to any cause in 


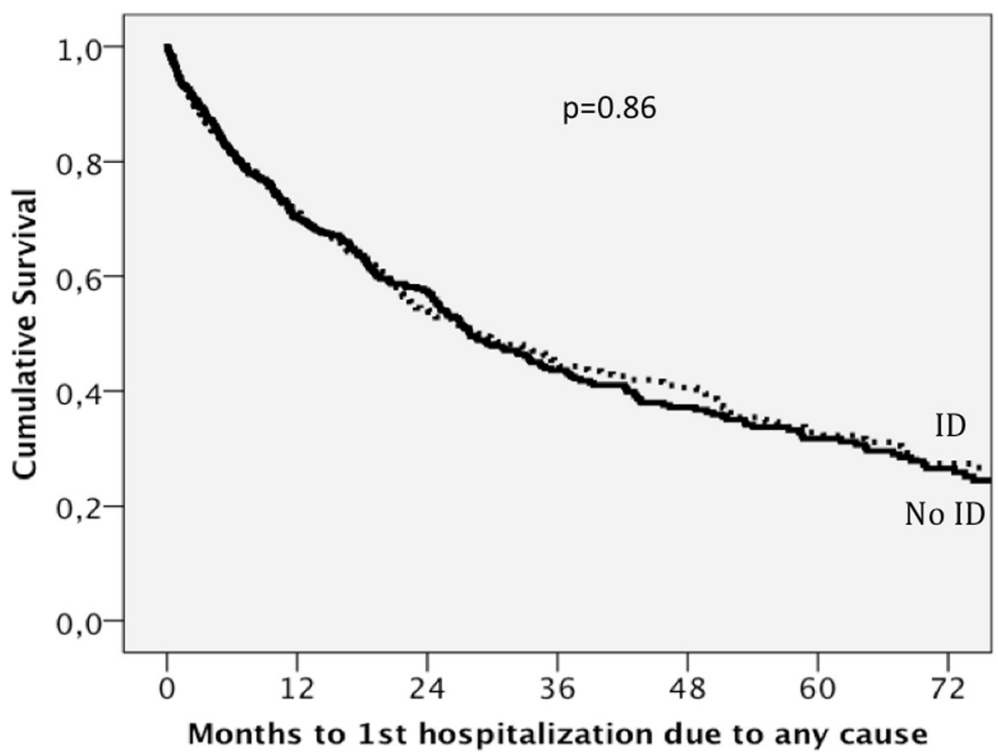

Fig. 4 Survival curves for hospitalization due to any cause stratified for ID after adjustment for the covariates that are significant in the multivariate model: Age, diabetes mellitus, chronic pulmonary obstructive disease, heart failure with preserved ejection fraction, New York Heart Association class III-IV, heart rate, haemoglobin, logarithmic transformation of N-terminal pro-brain natriuretic peptide, and treatment with an angiotensin converting enzyme inhibitor or angiotensin II receptor blocker, beta blockers and diuretics

univariate analysis, but again not after multivariate analysis. Predictors of hospitalization due to any cause were multiple and included all that predicted mortality and all that predicted hospitalizations due to HF, except for female sex and serum sodium, but with the addition of heart rate. What stands out from our analysis is that HFPEF was associated with a higher risk of hospitalizations due to heart failure or to any cause, and this may be related not so much to heart failure itself but to the increased cardiovascular and non-cardiovascular comorbidities of patients with HFPEF [21]. One comorbidity that is particularly prevalent in HFPEF is chronic pulmonary obstructive disease [22], and it is notable that in our cohort it was strongly associated with increased hospitalizations due to HF and to any cause but not with mortality, as has been shown in previous studies of patients with HF [23, 24].

It is plausible that in our cohort, other factors might diminish the importance of ID as a predictor of hospitalizations. However, our patient population clearly resembles the usual patients seen in clinical practice [18] and our results issue a word of caution in extrapolating that ID is linked with increased hospitalizations either due to HF or to any cause. Therefore, further studies are needed to evaluate this association, and in this direction, a report linking ID with 30 days readmission in patients with acute heart failure was recently published [25].

\section{Iron deficiency in non-anaemic patients}

In the FAIR-HF trial, treatment of ID with ferric carboximaltose in patients with chronic HF was equally efficacious in anaemic and in non-anaemic patients [26]. Given the fact that in our study, anaemia but not ID predicted mortality, we explored the association of ID with prognosis in non-anaemic patients. Although ID predicted increased mortality and hospitalizations in univariate analysis, this association did not hold up in multivariate analysis.

\section{Limitations}

We did not measure other markers of ID, such as the soluble transferrin receptor, hepcidin or the ferritin index that may better evaluate iron metabolism in patients with chronic heart failure [5, 27, 28]. We also did not assess $\mathrm{C}$-reactive protein, high-sensitivity cardiac troponin $\mathrm{T}$, and high-sensitivity soluble $\mathrm{ST} 2$, which have been associated with increased mortality in patients with chronic HF [3, 15]. We had no information regarding other non-cardiovascular comorbidities such as cancer or liver disease that could be potential confounders of outcomes in such an elderly population.

We excluded from the analysis patients that received treatment with intravenous iron or erythropoietin, creating a possible bias. However, this treatment was not approved by the guidelines at the time the study was performed and given that both these treatments could influence ID, we preferred to exclude these patients.

When assessing hospitalizations we did not look at recurrent number of hospitalizations, and this is also an important outcome that influences quality of life in patients with HF. 


\section{Conclusions}

In a contemporary cohort of patients with chronic HF, ID was present in more than half of the patients. However, its presence was not a predictor of mortality or hospitalizations due to HF or to any cause after multivariate adjustment. Further studies are needed to assess the prognostic significance of ID in real life patients with chronic HF and where HFPEF is increasingly prevalent.

\section{Abbreviations}

CKD: Chronic kidney disease; HF: Heart failure; HFPEF: Heart failure with preserved ejection fraction; ID: Iron deficiency; NT-proBNP: N-terminal pro-brain-type natriuretic peptide; TSAT: Transferrin saturation index

\section{Acknowledgments}

We would like to thank Dr. Julián Rodríguez Larrea for support in performing the statistical analysis and the CERCA Programme/Generalitat de Catalunya for institutional support.

\section{Funding}

The design and collection of the data by the investigators from Hospital Germans Trias i Pujol was supported by Red de Investigación Cardiovascular - RIC (RD12/0042/0047) and Fondo de Investigación Sanitaria, Instituto de Salud Carlos III (FIS PI14/01682) projects as part of the Plan Nacional de I+D + I and cofounded by ISCIII-Subdirección General de Evaluación y el Fondo Europeo de Desarrollo Regional (FEDER).

\section{Availability of data and materials}

The datasets used and/or analyzed during the current study are available from the corresponding author on reasonable request.

\section{Authors' contributions}

JGC, JCC, JL and ABG devised the study and developed it. JGC, CE, MDA, LF, $\mathrm{PMB}, \mathrm{NF}$ and EZ collected data, JGC analyzed the data and wrote the manuscript. JCC, JL, NM, RP and ABG contributed to the manuscript review process, including analysis and interpretation of the data and provided significant ideas for the execution of the study. All authors critically reviewed the manuscript for important intellectual content, approved the final manuscript and agree to be accountable for all aspects of the work.

\section{Ethics approval and consent to participate}

Written informed consent was obtained from each patient. The investigation conforms with the principles outlined in the Declaration of Helsinki. The local ethic committee from the Hospital Universitari de Bellvitge approved the study, ref:: PR088/16.

\section{Consent for publication}

Not applicable.

\section{Competing interests}

JCC has received speaking fees from Vifor Pharma and was a member of the steering committee of the FAIR-HF and CONFIRM-HF studies. None of the other authors have any conflicts of interest.

\section{Publisher's Note}

Springer Nature remains neutral with regard to jurisdictional claims in published maps and institutional affiliations.

\section{Author details}

${ }^{1}$ Area de Enfermedades del Corazón, Hospital Universitari de Bellvitge, IDIBELL, Universitat de Barcelona, L'Hospitalet de Llobregat, Feixa Llarga SN, 08907 Barcelona, Spain. '2Servicio de Cardiología, Hospital del Mar, IMIM, Universitat Autònoma de Barcelona, Barcelona, Spain. ${ }^{3}$ Unidad de Insuficiencia Cardíaca, Hospital Universitari Germans Trias i Pujol, Universitat Autònoma de Barcelona, Badalona, Barcelona, Spain. ${ }^{4}$ Servicio de Medicina Interna, Hospital Universitari de Bellvitge, IDIBELL, University of Barcelona, L'Hospitalet de Llobregat, Barcelona, Spain.
Received: 2 April 2018 Accepted: 19 October 2018

Published online: 01 November 2018

\section{References}

1. Jankowska EA, Rozentryt P, Witkowska A, Nowak J, Hartman O, Ponikowska $B$, et al. Iron deficiency: an ominous sign in patients with systolic chronic heart failure. Eur Heart J. 2010;31:1872-80.

2. Klip IT, Jankowska EA, Enjuanes C, Voors AA, Banasiak W, Bruguera J, et al. The additive burden of iron deficiency in the cardiorenal-anaemia axis: scope of a problem and its consequences. Eur J Heart Fail. 2014;16:655-62.

3. Parikh A, Natarajan S, Lipsitz SR, Katz SD. Iron deficiency in communitydwelling US adults with self-reported heart failure in the National Health and nutrition examination survey III. Prevalence and association with anaemia and inflammation. Circ Heart Fail. 2011:4:599-606.

4. González-Costello J, Comin-Colet J. Iron deficiency and anaemia in heart failure: understanding the FAIR-HF trial. Eur J Heart Fail. 2010;12:1159-62.

5. Jankowska EA, Malyszko J, Ardehali H, Koc-Zorawska E, Banasiak W, von Haehling $S$, et al. Iron status in patients with chronic heart failure. Eur Heart J. 2013;34:827-34.

6. Haddad S, Wang Y, Galy B, Korf-Klingebiel M, Hirsch V, Baru AM, et al. Ironregulatory proteins secure iron availability in cardiomyocytes to prevent heart failure. Eur Heart J. 2017;38:362-72.

7. Lewis GD, Malhotra R, Hernandez AF, McNulty SE, Smith A, Felker M, et al. Effect of oral iron repletion on exercise capacity in patients with heart failure with reduced ejection fraction and iron deficiency. The IRONOUT HF randomized clinical trial. JAMA. 2017;317:1958-66.

8. Anker SD, Colet JC, Filippatos G, Willenheimer R, Dickstein K, Drexler H, et al. Ferric carboxymaltose in patients with heart failure and iron deficiency. $\mathrm{N}$ Engl J Med. 2009;361:2436-48.

9. Ponikowski P, van Veldhuisen DJ, Comin-Colet J, Ertl G, Komajda M, Mareev $V$, et al. Beneficial effects of long-term intravenous iron therapy with ferric carboxymaltose in patients with symptomatic heart failure and iron deficiency. Eur Heart J. 2014;36:657-68.

10. Jankowska EA, Tkaczyszyn M, Suchocki T, Drozd M, von Haeling S, Doehner $W$, et al. Effects of intravenous iron therapy in iron-deficient patients with systolic heart failure: a meta-analysis of randomized controlled trials. Eur J Heart Fail. 2016;18:786-95.

11. Van Veldhuisen DJ, Ponikowski $P$, van der Meer P, Metra M, Bohm M, Doletsky $A$, et al. Effect of ferric carboxymaltose on exercise capacity in patients with chronic heart failure and iron deficiency. Circulation. 2017;136: 1374-83.

12. Ponikowski P, Voors AA, Anker SD, Bueno H, Cleland JGF, Coats AJS, et al. Guidelines for the diagnosis and treatment of acute and chronic heart failure. The task force for the diagnosis and treatment of acute and chronic heart failure of the European Society of Cardiology. Developed with the special contribution of the heart failure association (HFA) of the ESC. Eur Heart J. 2016;37:2129-200.

13. Wu A. Tietz Clinical Guide to Laboratory Tests. 4th ed. St Louis, MO: Saunders Elsevier; 2006. p. 56-61.

14. Klip IT, Comin-Colet J, Voors AA, Ponikowski P, Enjuanes C, Banasiak W, et al. Iron deficiency in chronic heart failure: an international pooled analysis. Am Heart J. 2013;165:575-82.

15. Lupón J, de Antonio M, Vila J, Peñafiel J, Galán A, Zamora E, et al. Development of a novel heart failure risk tool: the Barcelona bio-heart failure risk calculator (BCN bio-HF calculator). PLoS One. 2014;9:e85466.

16. Jankowska EA, Kasztura M, Sokolski M, Bronisz M, Nawrocka S, OleskowskaFlorek W, et al. Iron deficiency defined as depleted iron stores accompanied by unmet cellular iron requirements identifies patients at the highest risk of death after an episode of acute heart failure. Eur Heart J. 2014;35:2468-76.

17. Fang J, Mensah GA, Croft JB, Keenan NL. Heart failure-related hospitalization in the U.S., 1979 to 2004. J Am Coll Cardiol. 2008;52:428-34.

18. Berry C, Murdoch DR, McMurray JJ. Economics of chronic heart failure. Eur J Heart Fail. 2001;3:283-91.

19. Comin-Colet J, Enjuanes C, Gonzalez G, Torrens A, Cladellas M, Meroño O, et al. Iron deficiency is a key determinant of health-related quality of life in patients with chronic heart failure regardless of anaemia status. Eur J Heart Fail. 2013;15:1164-72.

20. Crespo-Leiro MG, Segovia-Cubero J, González-Costello J, Bayes-Genis A, López-Fernández S, Roig E, et al. Adherence to the ESC Heart Failure Treatment Guidelines in Spain: ESC Heart Failure Long-term Registry. Rev Esp Cardiol. 2015;68:785-93. 
21. Lund LH, Donal E, Oger E, Hage C, Persson H, Haugen-Löfman I, et al. Association between cardiovascular vs. non-cardiovascular co-morbidities and outcomes in heart failure with preserved ejection fraction. Eur J Heart Fail. 2014:16:992-1001.

22. Iversen KK, Kjaergaard J, Akkan D, Kober L, Torp-Pedersen C, Hassager C, et al. Chronic obstructive pulmonary disease in patients admitted with heart failure. J Intern Med. 2008;264:361-9.

23. Rusinaru D, Saaidi I, Godard S, Mahjoub H, Battle C, Tribouilloy C. Impact of chronic obstructive pulmonary disease on long-term outcome of patients hospitalized for heart failure. Am J Cardiol. 2008;101:353-8.

24. Hawkins NM, Huang Z, Pieper KS, Solomon SD, Kober L, Velazquez EJ, et al. Chronic obstructive pulmonary disease is an independent predictor of death but not atherosclerotic events in patients with myocardial infarction: analysis of the valsartan in acute myocardial infarction trial (VALIANT). Eur J Heart Fail. 2009;11:292-8

25. Núñez J, Comín-Colet J, Miñana G, Núñez E, Santas E, Mollar A, et al. Iron deficiency and risk of early readmission following hospitalization for acute heart failure. Eur J Heart Fail. 2016;18:798-802.

26. Filippatos G, Farmakis D, Comin Colet J, Dickstein K, Lüscher TF, Willenheimer $R$, et al. Intravenous ferric carboxymaltose in iron deficient chronic heart failure patients with and without anaemia: a subanalysis of the FAIR-HF trial. Eur J Heart Fail. 2013;15:1267-76.

27. Jankowska EA, von Haehling S, Anker SD, Macdougall IC, Ponikowski P. Iron deficiency and heart failure: diagnostic dilemmas and therapeutic perspectives. Eur Heart J. 2013;34:816-29.

28. Cullis JO. Diagnosis and management of anaemia of chronic disease: current status. Br J Haematol. 2011;154:289-30.

Ready to submit your research? Choose BMC and benefit from:

- fast, convenient online submission

- thorough peer review by experienced researchers in your field

- rapid publication on acceptance

- support for research data, including large and complex data types

- gold Open Access which fosters wider collaboration and increased citations

- maximum visibility for your research: over $100 \mathrm{M}$ website views per year

At BMC, research is always in progress.

Learn more biomedcentral.com/submissions 